\title{
AC 2009-2193: TEACHING SOCIAL COMPLEXITY AND MULTIDISCIPLINARY TEAM BUILDING: AN EXPERIMENTAL ENGINEERING APPROACH
}

Craig Laramee, State University of New York, Binghamton

Shelley Dionne, State University of New York, Binghamton

Hiroki Sayama, State University of New York, Binghamton

David Wilson, State University of New York, Binghamton 


\title{
Teaching Social Complexity and Multidisciplinary Team Building: An Experimental Engineering Approach
}

\begin{abstract}
Numerous organizations, including the National Science Foundation (NSF), the Accreditation Board for Engineering and Technology (ABET), and the National Academy of Engineering have placed an emphasis on the need to better prepare engineering and science professionals to work successfully within the team-based structures of today's organizations. Additionally, engineering curriculum experts have called for the inclusion of leadership and managerial decision making training for engineers within graduate and undergraduate programs. To address this need, we have developed lecture and laboratory material that directly examines the social and political aspects of engineering through the use of experimentation, data analysis, and computer simulation.

This material was used in a new interdisciplinary junior level course with approximately 30 students from a wide range of disciplines. Using a modular format we examined concepts of collective intelligence, evolutionary product design and problem solving, game theory, collaborative problem solving, and social networks. The understanding of these concepts was reenforced using interactive simulations and game play with immediate feedback and discussion. This was facilitated using a hand held wireless computer mediated communication (CMC) system that enabled synchronous participation of students in traditional decision theory games and exercises. This system allowed us to capture, in real time, individual, team, and class level outcomes that assisted in the evaluation of important considerations in complex social systems and better reinforcement of group dynamic theory and leader emergence.
\end{abstract}

Preliminary results indicate that using this material, students developed a better understanding of the factors that lead to effective multidisciplinary teams. Additionally, analysis of student feedback identified key areas to improve laboratory exercises but also suggests that students find the activity periods not only enjoyable but also effective at developing a deeper understanding of concepts.

\section{Introduction}

Organizations around the globe have increased their reliance and dependence on work teams to provide solutions to problems that are too complex for individuals to solve alone ${ }^{1,2,3,4}$. Indeed, a recent report from the National Academy of Engineering highlighted the importance of a systems engineering perspective for contemporary engineering challenges ${ }^{5}$. This perspective inherently requires a broad range of skills so that broader requirements can be met. These include:

Complexity: Engineers must know how and when to incorporate social elements into a comprehensive systems analysis of their work.

Customerization: Increasingly, engineers will have to interact with customers demanding that engineers have well-developed people skills, that is, an understanding of how different cultures, goals and attitudes influence the decision process. 
Public Policy: The roles that engineers take on have always extended beyond the realm of knowledge and technology. As technology becomes increasingly engrained into every facet of our lives, the convergence between engineering and public policy also will increase.

As the scale and complexity of systems based engineering problems expands in a technologically advanced global business world, decision making has become increasingly complex, with a growing need to pursue collaborations with interdisciplinary teams of experts across multiple fields. Essential to the effectiveness of these teams is an understanding of their dynamics and emergent properties. For example, high levels of teamwork and employee interdependence have resulted in self-managed work teams, participative decision making, and extensive knowledge of team processes to promote effective and sustainable organizational productivity ${ }^{2,6}$.

In this paper we report on our development of educational materials that aim to directly address the social and political aspects of engineering through the use of experimentation, data analysis and computer simulation.

\section{Course Materials}

Our initial offering of this material took place in the fall semester of 2008 in a junior level course offered at Binghamton University that was cross-listed across three schools, the Thomas J. Watson School of Engineering in the department of Bioengineering, the Harpur College of Arts and Sciences in the department of Biology and in the School of Management. This course was co-taught by four instructors, two from Bioengineering, and one each from the other departments. Throughout this course, students from these traditionally distinct disciplines used a computer mediated communication (CMC) system to participate in simulations and game play that highlighted concepts in organizational and social behavior.

The CMC system consisted of approximately 30 handhelds (either Dell Axim x30 Pocket PC's or iPod touch) that wirelessly connected to a web server which provided the simulation software, collected simulation data, and performed statistical analysis of outcomes (figure 1).

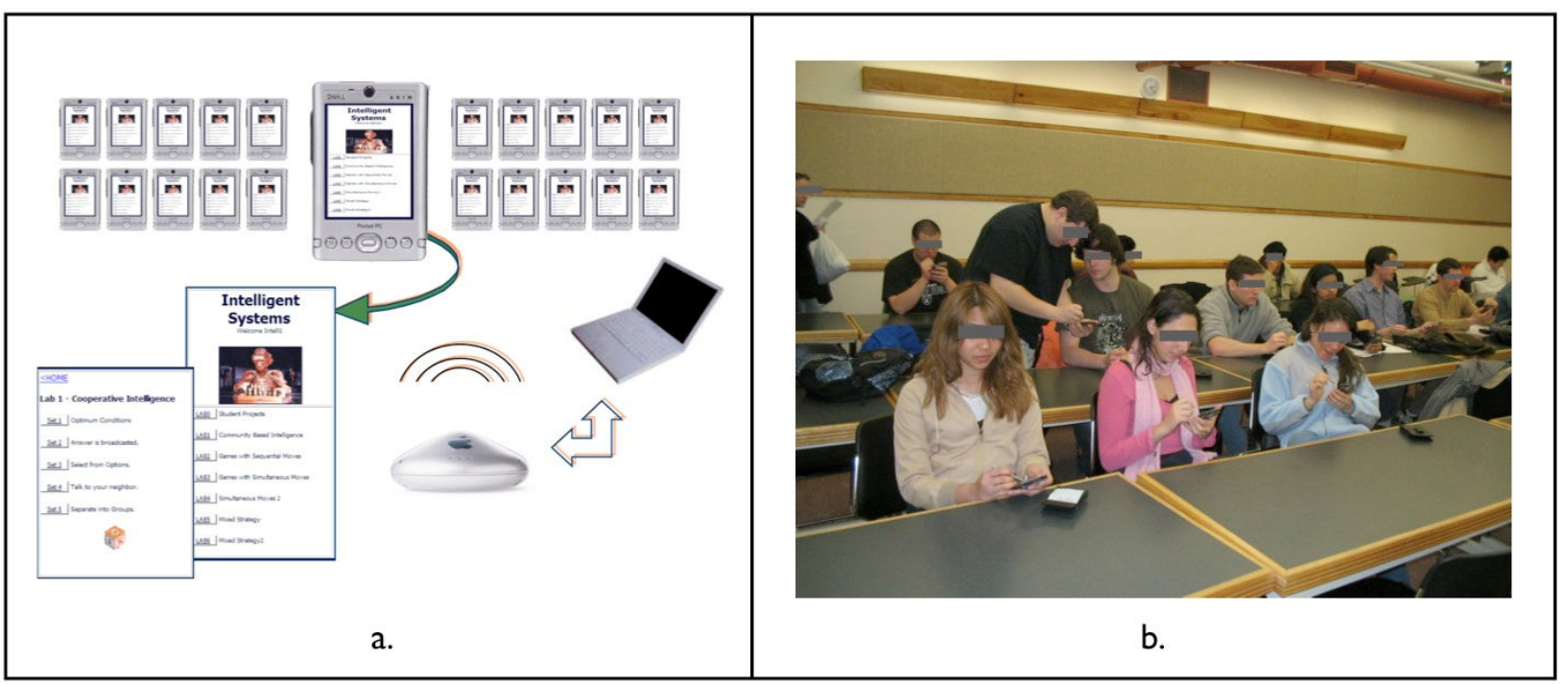


Figure 1: a.) The CMC-based system to enable active student participation in simulations and real-time collection and presentation of individual, group, and class level outcomes. b.) Students using the handhelds participating in a simulation.

This system was designed to be transparent to the user such that the only requirement for the handhelds was that they be able to connect to the internet, and no programming was necessary for the students. Our goal was to create an active learning environment where students could iteratively formulate hypotheses, perform experiments, and analyze the emergent social and behavioral outcomes in real time.

The course material was organized info five modular units (2-3 weeks per unit) that consisted of lectures followed by activity periods and terminated with group final projects. In each of these modules, topics and activities were chosen to examine concepts of leadership, teamwork and other emergent social behaviors. They included the following:

Collaborative Problem Solving: Problem solving skills in a collaborative team-based environment are among the most important skill sets that engineers in the 21 st century must have. This module helps students understand potentials and limitations of collaborative problem solving processes. Students are given nontrivial multifaceted engineering-related (but not technically specific, to remain relevant for the non-engineering team members) problems that require creative approaches.

Lecture: Discussion of collaborative problem solving using lectures and examples from primary literature. Topics included fundamental theoretical principles relevant to the functioning and malfunctioning of groups from an evolutionary perspective and principles relevant to human social behavior including, in particular, principles relevant to human problem solving behavior as a particular kind of social interaction.

Lab: Students solve problems based on published literature comparing the efficacy of individuals vs. groups in solving a complex cognitive task. Some work individually while others work in groups then individual and group performances are statistically evaluated and compared. This process is repeated with different experimental parameters enabling students to learn communication and motivation strategies needed to make groups function effectively. Students also examine how collaborative problem solving can produce more innovative solutions, and limitations that exist within group contexts. An example activity is shown in figure 2. 


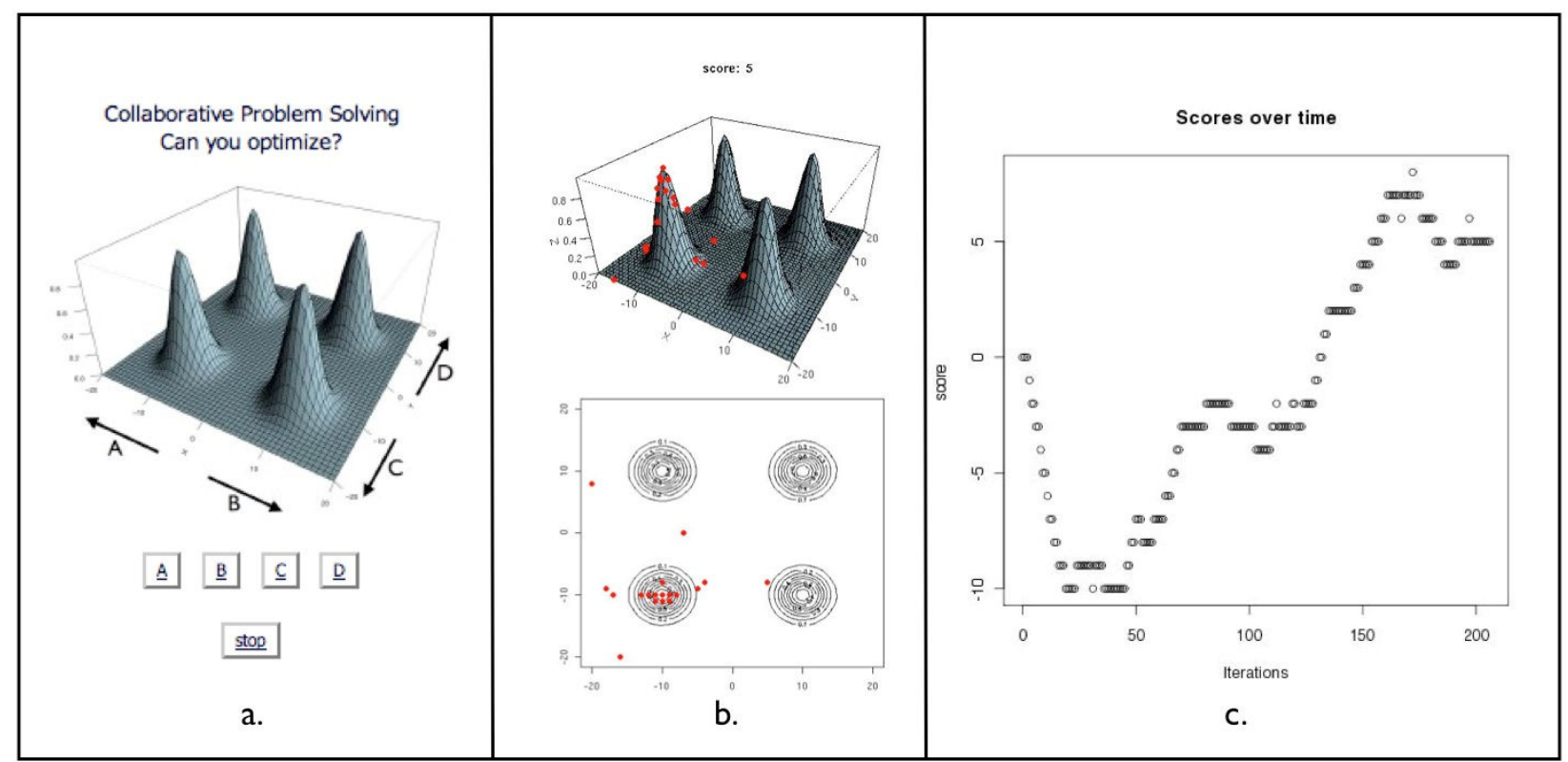

Figure 2: A hill climbing activity that requires students to work together to maximize the collective score. a.) A screenshot of what the students see on the handheld device. b.) a screenshot of what is displayed at the front of the room. Each student is represented by a red dot which is updated in real time. c.) Collective scores are displayed after simulation. These scores correlated to the social dynamics that took place among the students. When the students worked together they achieved better performance and the collectie score increased, when the collaboration broke down so did group performance and the collective score decreased. Several cycles of this can be seen in this figure.

Collective Intelligence: Collective intelligence can be defined as groups of individuals doing things collectively that seem intelligent, and is highly relevant to modern society. Moreover, the formation and self-organization of collective knowledge through computer-mediated networks is now pervasive and extensively used in engineering and many other fields. This module helps students understand how such collective intelligence may (or may not) work and how one can exploit its potential for specific problem solving. Students are given questions to answer (e.g. how many miles is it from Atlanta to Binghamton?) and observe the emergent property of collective intelligence, how groups can "know" more than any one individual in the group.

Lecture: Discussion of collective intelligence and an understanding of the concepts, models and issues of Collective Intelligence (CI) is accomplished through readings and lectures. Specific forms of CI (e.g. reflexive, structural, evolutionary, statistical, etc.) as well as models and methods to achieve CI (e.g. Delphi technique) are examined.

$L a b$ : Students use the CMC system to provide answers to several sets of questions (how many miles is it from Atlanta to New York? What is the height in feet of the Great Pyramid in Egypt? Etc.) Responses are examined at the individual (anonymous), group and class level demonstrating how a group can "know" more than any one individual in the group. This experiment is repeated with different sets of questions and different constraints that can affect the collective behavior of the group. For example, removing anonymity, removing parallelism by having a "leader" announce their decision first, removing diversity by separating into groups e.g. male/female, etc. 
The impacts of these factors on group performance, team dynamics, and leadership are reviewed immediately after the simulations. Additionally, students are able to propose design constraints to alter group performance in a desired way which can immediately be implemented and tested. An example activity is shown in figure 3.

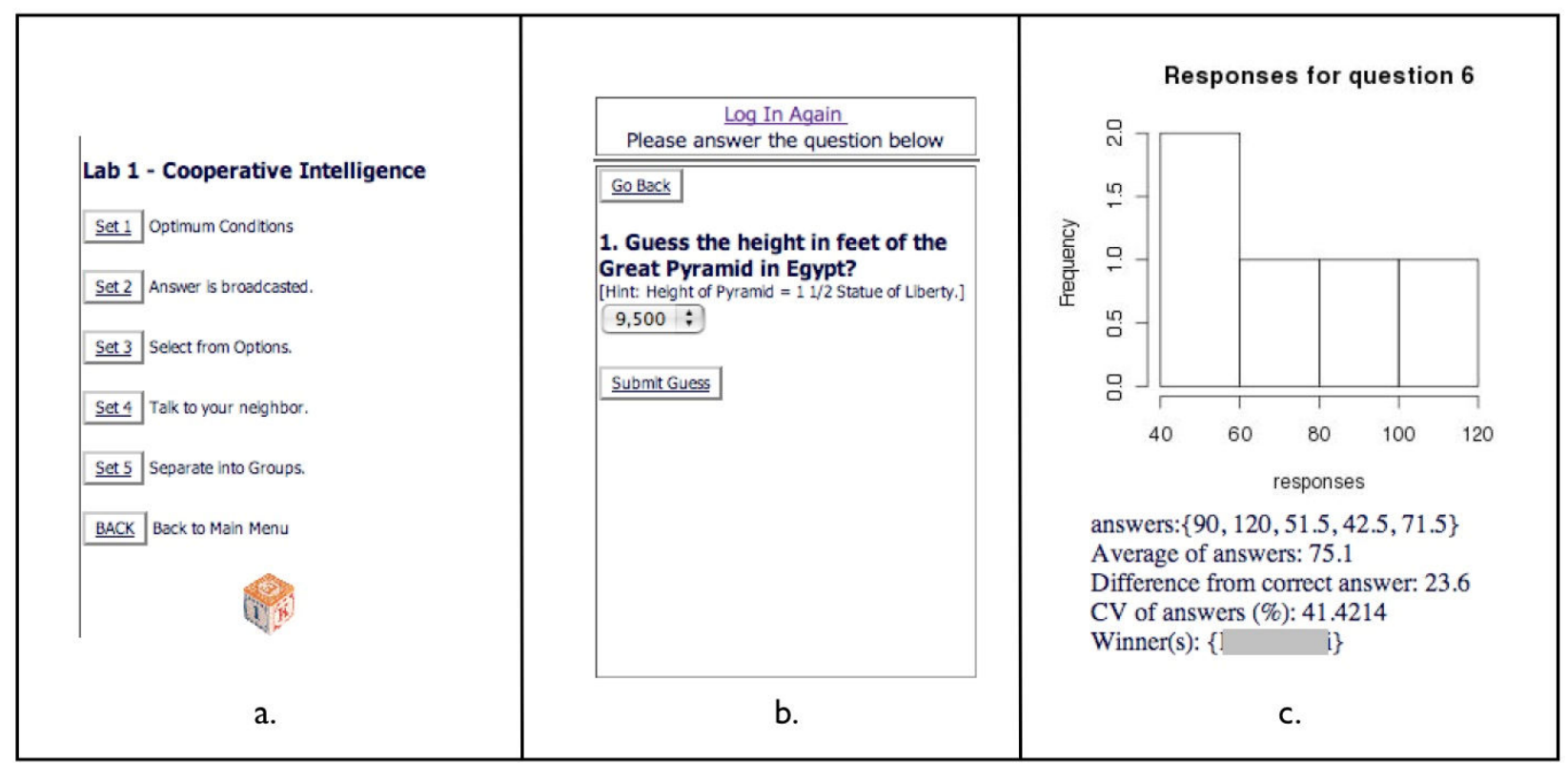

Figure 3: A collective intelligence activity that requires students answer a series of questions under different experimental constraints. a.) A screenshot of what the students see on the handheld device displaying the different experiments in the activity. b.) A screenshot of what a student would see during one of the experiments. In this case the set of answers is constrained by using a dropdown menu. c.) Distribution of answers and aggregated collective answers are displayed after simulation. These emergent outcomes are discussed in the context of the social dynamics that took place during the activity and the factors that give rise to collective intelligence.

Game Theory: Virtually almost all the critical problems related to science and technology that humanity is facing today (e.g., global warming, environmental pollution, population explosion, proliferation of nuclear weapons, etc.) arise from some kind of conflicts of interest between multiple parties involved in the problem. To develop an effective technical solution for such problems one must understand and exploit dynamics of social conflicts in various settings, for which game theory provides a very useful framework. This module introduces students to the concepts of game theory and lets them participate in simulations to investigate strategic "behaviors" including altruism, satisficing, rational choice theory and the impact of social interaction on situations with both (or either) rational decision making and naturalistic decision making.

Lecture: Discussion of game theoretic concepts including sequential and simultaneous games, mixed strategies, Nash equilibrium, informational asymmetries and the cognitive elements that play a role in decision making

$L a b$ : Students play games of strategy including the Trustee/Investor game and the Full 
Information Aces and Deuces game to examine the concepts of Self Serving Bias and Pre-play Communication. Students also use the CMC system to participate in interactive game simulations including Prisoners Dilemma and the Ultimatum game where strategic behaviors are examined under different conditions such as removing opponent anonymity, and providing information (e.g. revealing opponents scores, tendencies, etc.). The impacts of these factors on strategic behaviors as well as social behaviors (vocal manipulations, revenge, tit-for-tat, etc.) are reviewed immediately after simulations facilitating discussion while students emotional states remain relevant. An example activity is shown in figure 4.

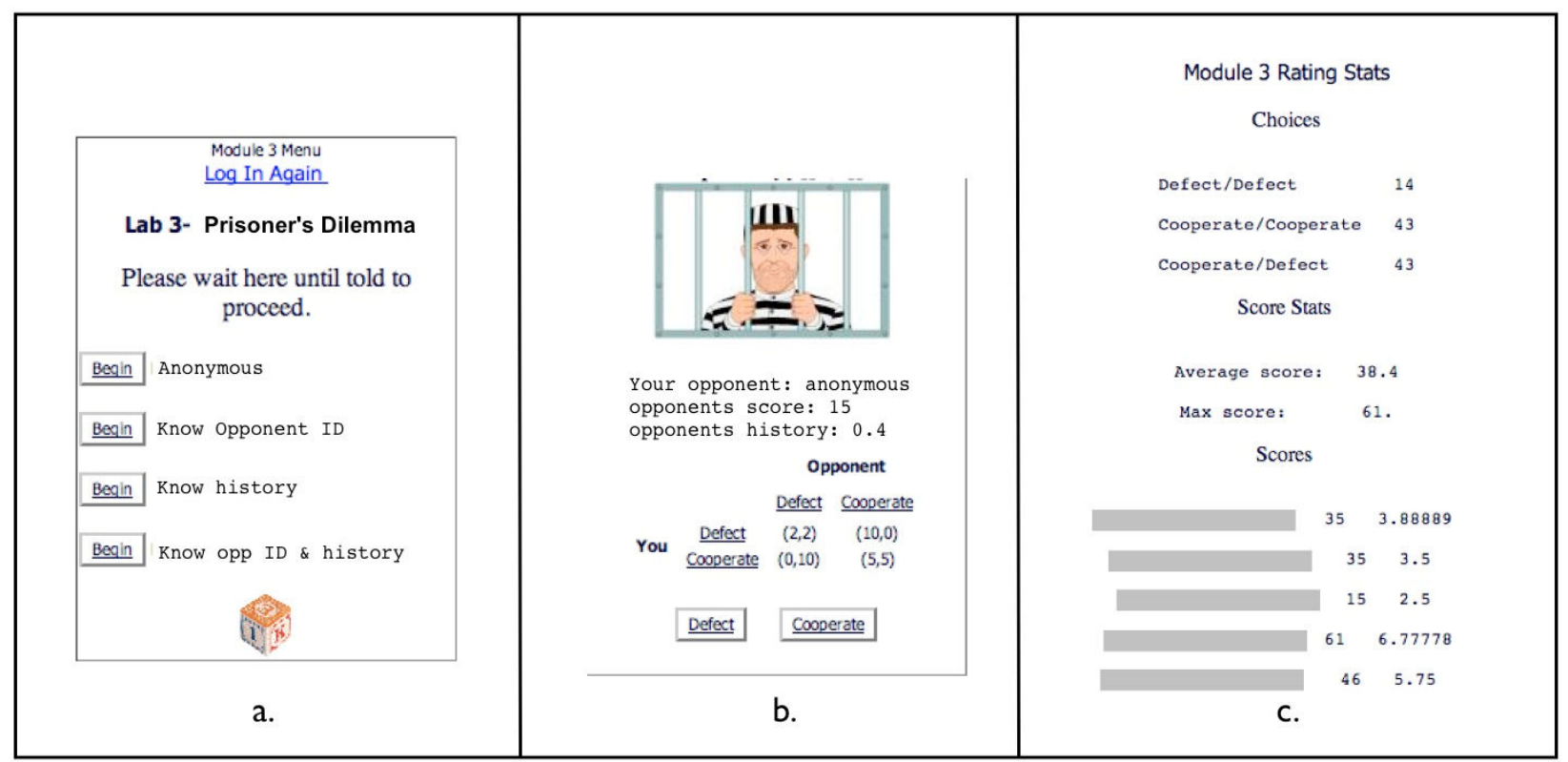

Figure 4: A game theory activity that enables students to participate in different games of prisoners dilemms. a.) A screenshot of what the students see on the handheld device displaying the different experiments in the activity. b.) A screenshot of what a student would see during one of the experiments. In this case each student has knowledge of their opponents tendency to cooperate and their score. c.) Distribution of strategies played along with group average scores and individual scores are displayed after simulation. These outcomes are immediately discussed after each experiment and related to the social dynamics that took place during that experiment.

Evolutionary Product Design and Problem Solving: Evolution is a powerful conceptual tool that can explain dynamical changes in various systems by combining replication, variation and selection of entities. Because of its broad applicability, understanding evolution is now as important in engineering and social sciences as in biology where it was originally developed. This module introduces students to the basics of evolutionary theory and helps them learn how to apply evolutionary processes to product design and problem solving by participating in the simulations.

Lecture: Discussion of natural selection in the context of evolutionary product design and problem solving. Topics include mechanisms of informational exchange such as linear vs. nonlinear and symmetric vs. asymmetric influences, and their applications to product design and problem solving, including, evolutionary computation and interactive evolutionary methods. 
Lab: Students participate in the evolution of "information" among students in a classroom and to observe the evolutionary dynamics in real time. Examples include the design of some product (e.g., a motto, a public statement, or a graphical logo), each of which can be encoded in a chromosomal representation. Replication and variation are achieved by copying information from one selected design to another with some mutation and crossover operations. Selection is achieved by built-in selection criteria and students' subjective choices. Experiments are carried out with several different settings, enabling students to learn how evolution works and see the impact that an evolutionary design approach can have in social and engineering contexts. An example activity ${ }^{7}$ is shown in figure 5 .

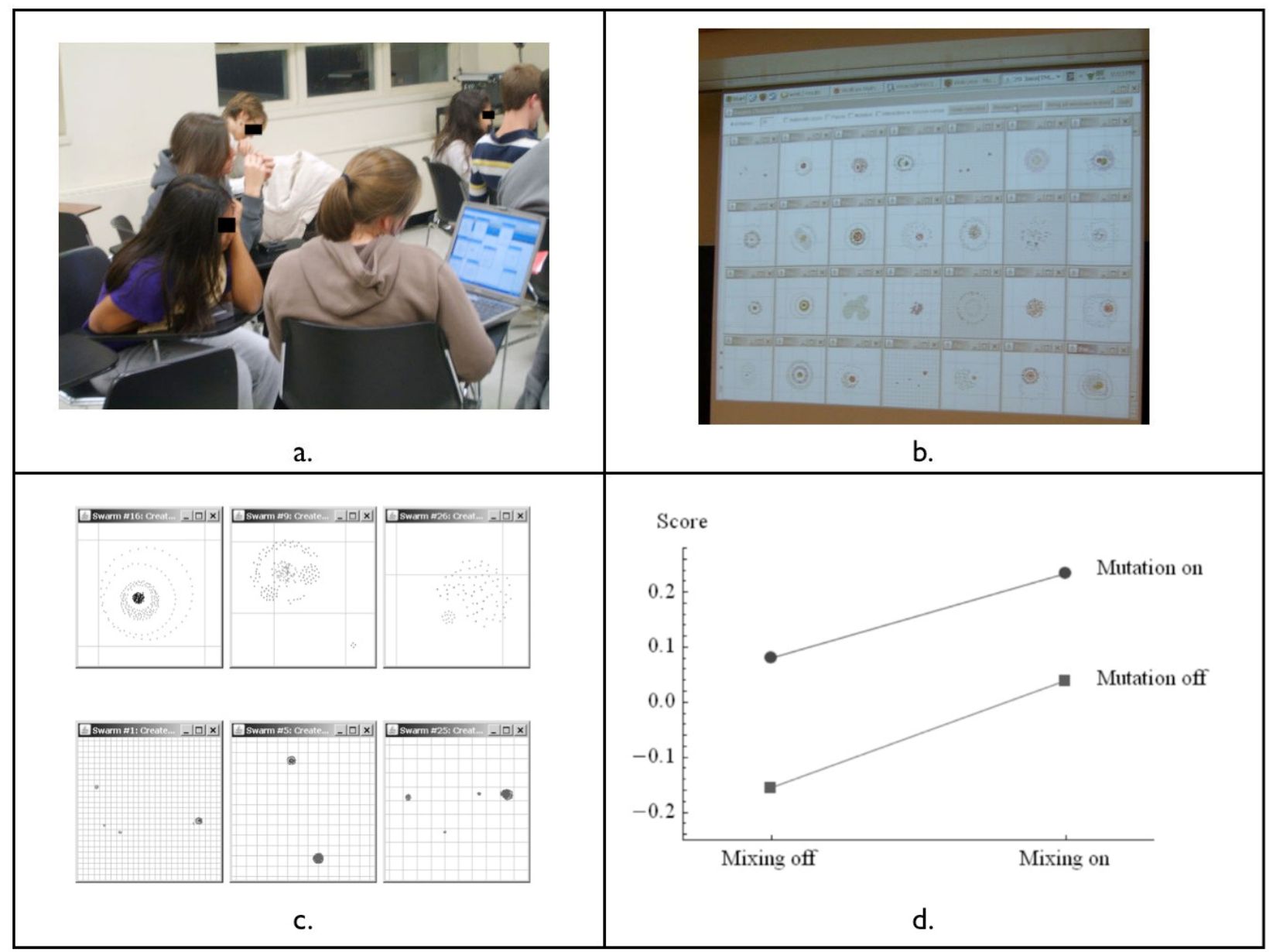

Figure 5: Using a Swarm Chemistry simulator, students participated as designers and then evaluators of swarms. The objective of this experiment was to quantitatively evaluate how each of the evolutionary operators improved the overall "quality" of outcomes of evolutionary design. a.) Particle swarms are generated by students working in groups using one of four evolutionary strategies (1: neither mixing nor mutation operators, 2: Mixing only, 3: Mutation only, 4: Mixing and mutation). b.) 28 swarms selected by the students are simultaneously simulated and projected on a large screen in the classroom for students' peer evaluation. c.) Samples of the final swarm designs created by students. Top: The three that received the highest rating scores. Bottom: The worst three that received the lowest rating scores. d.) Mean normalized scores plotted with regard to two sources of variation (mixing on/off and mutation on/off). 
Social Networks: Properties of large-scale complex networks commonly seen in biological, social and engineered systems have recently been of particular interest in many fields of science and engineering, especially because of their rich implications for our societal structures and dynamics. Many of the today's successful examples of technological solutions have exploited the nature of such complex networks, and therefore, engineers in the 21 st century must be aware of the prevalence of networks in almost every scene of our life and their fundamental properties. This module introduces students to the rapidly developing science of networks and helps them understand how social networks grow, self-organize, and acquire their unique properties that are distinct from those of random or uniformly connected manmade networks.

Lecture: Discussion of recent findings in network science, including exponential, small-world, and scale-free networks, several known mechanisms that lead to the formation of such networks, and the topological/dynamical properties of those networks. Ego-centric as well as networkcentric metrics such as degree distribution and centrality measures are examined in the context of information flow and social structures.

$L a b$ : Students will use the CMC system to create a relational network in real time within the class by observation of communication networks among students given a problem to solve. This network is then examined for social structures and information flows and compared with group performances. Students then utilize their knowledge of network properties in a competition to perform a "state flip" of three different types of networks that contain characteristics observed in real social networks. An example activity is shown in figure 6.

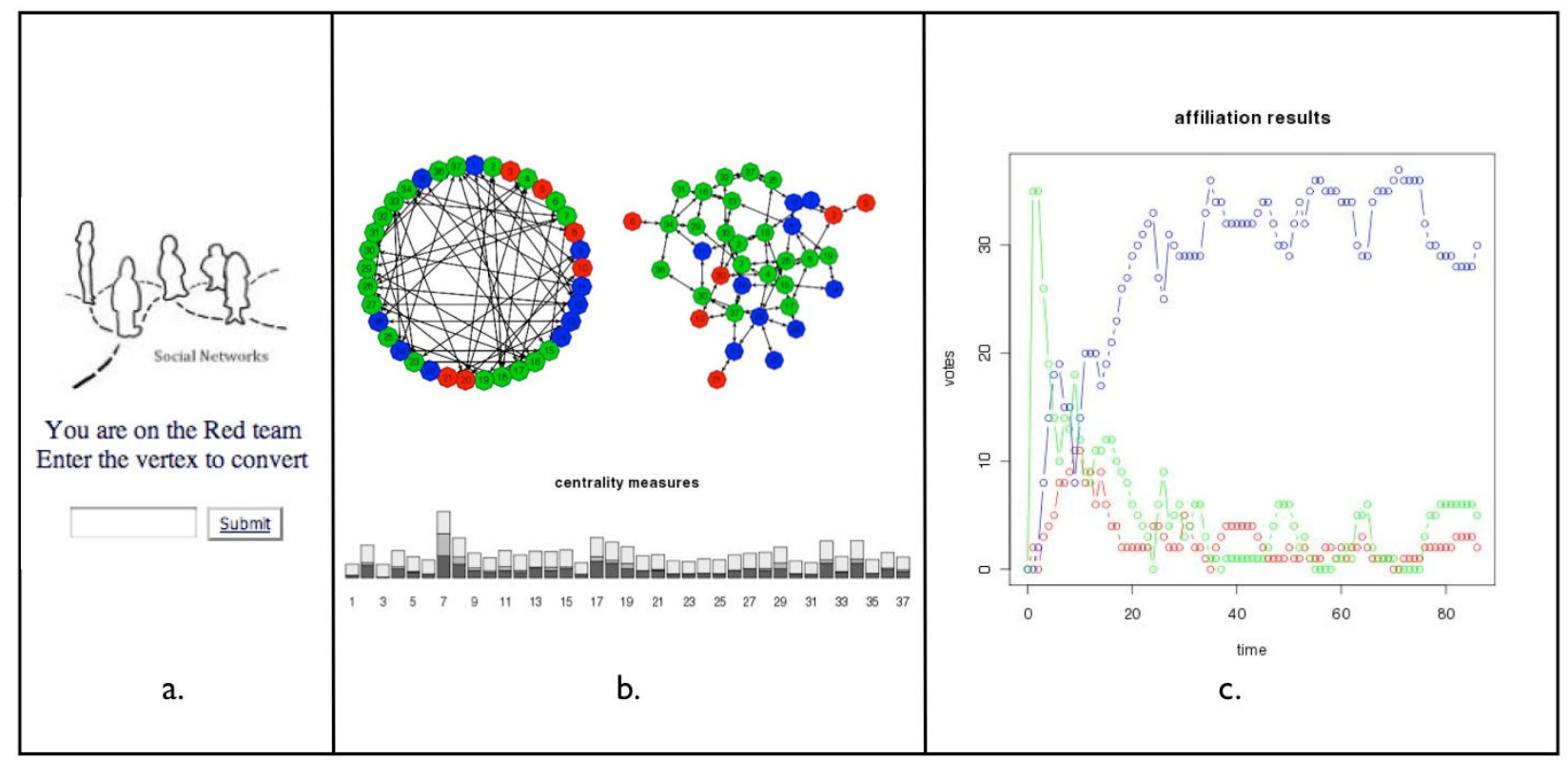

Figure 6: A social network activity in which students are selected into teams (red, blue) and are tasked with the objective of flipping the state of a connected network to their color. Each student selects a particular vertex to "win over" based on their knowledge of social networks and the strategy of their team. a.) A screenshot of what each student would see on their handheld. b.) A screen shot of what is projected at the front of the class. This shows the centrality scores of each of the vertices as well as the current state of each vertex and is updated in real time. c.) The overall network state is displayed after the simulation showing the dynamics of state changes as a 
function of time. This is is discussed in the context of the social dynamics that took place during the activity. In this figure it can be seen that the blue group worked more effectively as a team and was eventually able to flip the state of the network.

Final Projects: At the end of the course students had to utilize what they had learned in final projects. For these projects students were assigned into groups of approximately 3-4 individuals and given the problem to develop a simulation (game, etc.) that examines at least one of the aspects of social dynamics that has been covered in this course. Specifically, each project had to contain the following items:

- Background research on the topic(s) that you are investigating.

- Experimental design of the proposed simulation including expected outcomes, identification of confounding factors, and appropriate analytical procedures. A computational simulation of expected outcomes may be helpful although not necessary.

- Conducting the simulation, data collection and computation of analytical results.

- Discussion and interpretation of the results.

Students gave a 10 minute presentation of their projects on the last day of class.

\section{Student Reactions and Future Modifications}

Overall the students were very responsive to the material presented and the activities conducted in this course. Learning was assessed using exams and essay based homework assignments and student feedback was obtained by having students write reaction papers to the activities immediately after they were performed. From this feedback, it was clear that the students enjoyed the activities and they provided valuable information on specific areas that each activity could be improved.

A key strength of the student performance throughout this course was the improvement in interaction skills among diverse students as the semester progressed. As students learned more about group dynamics (a component informally or formally addressed in each meeting session), interactions became more engaging, and peer-to-peer feedback became more prevalent. Additionally, due the high level of interactive exercises and activities in class, differences in opinions, approaches and problem solving directions were more apparent, and as the semester progressed, students solved these issues in a more immediate fashion. Conflict management between students would usually involve stopping small group proceedings and addressing key differences, restructuring the response, and continuing the group exercise with improved structure to the group process. Thus, imparting knowledge regarding social dynamics and group process was particularly effective.

A weakness in the learning outcomes was the level of sophistication students employed to illustrate their methods-based learning. Although all final projects used hypothesis development and testing through data collection and analysis, students used very basic analytic tools. More sophisticated testing and analysis was possible, and teams were structured by instructors in such a way as to maximize unique abilities of students, however, most teams underutilized their unique expertise.

The outcomes of this course also illustrate the benefit of using an active learning approach to 
deliver the concepts important for preparing students for the social and political aspects of engineering. Additionally, they support the use of technologies such as the wireless CMC system we developed for this course for rapid data collection and analysis. An additional benefit of this approach that was the direct role that students had in the design of the course as they were not only simulation participants but they also had roles as simulation designers and experimenters.

In future versions of the course we will directly assess the effectiveness of our educational material and teaching strategies by giving a questionnaire to a control group of students that will not be enrolled in the course. Members of the control group will consist of student participants that are of similar ages to those anticipated in the course (juniors and seniors), and within the departments of Bioengineering, Biology, and Management. The responses of this control group will be statistically compared to responses to similar questions from the students within the course to assess the effectiveness of this course in enhancing students understanding of social dynamics and complexity.

We would like to thank the National Science Foundation for their financial support in the development of this course material (NSF Award \# 0737313).

\section{Bibliography}

[1] Bettenhausen KL. Five years of groups research: What we have learned and what needs to be addressed. Journal of Management. 17: 345-381. 1991

[2] Salas E and Cannon-Bowers JA. The science of training: A decade of progress. Annual Review of Psychology. 52: 471-499. 2001

[3] Kerr NL and Tindale RS. Group performance and decision making. Annual Review of Psychology. 55: 623-655. 2004

[4] Wilson DS, Timmel J, and Miller R. Cognitive cooperation: when the going gets tough, think as a group. Human Nature. 15: 225-250. 2004

[5] National Academy of Engineering, National Academy of Sciences The Engineer of $2020 \mathrm{Đ}$ Visions of Engineering in the New Century. THE NATIONAL ACADEMIES PRESS Washington, DC. 2004 (http://books.nap.edu/catalog/10999.html)

[6] Fandt PM. The relationship of accountability and interdependent behavior to enhancing team consequences. Group and Organization Studies. 16: 300-312. 1991

[7] Sayama H, Dionne S, Laramee C, and Wilson DS. Enhancing the Architecture of Interactive Evolutionary Design for Exploring Heterogeneous Particle Swarm Dynamics: An In-Class Experiment. IEEE Symposium Series on Computational Intelligence 2009 